\title{
Ausdehnung eines Satzes vom ebenen Vierseit auf räumliche Figuren.
}

(Von Herrn O. Hermes.)

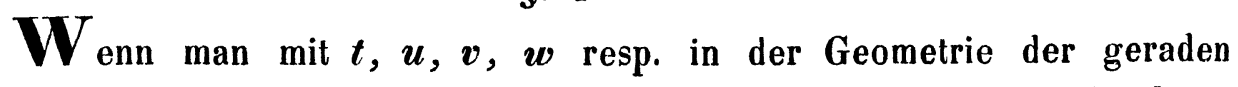
Linie, der Ebene und des Raumes die Abstände von zwei festen Punkten, von drei festen Geraden uud von vier festen Ebenen bezeichnet, so erhält man in der Geometrie der geraden Linie als Gleichungen eines harmonischen Punktsystems

$$
\boldsymbol{l t}+\boldsymbol{m u}=\mathbf{0}, \quad \boldsymbol{t} \boldsymbol{t}=\boldsymbol{m u} .
$$

Diesem System entspricht in der Geometrie der Ebene das System einer Geraden und eines Punktes, deren Gleichungen sind:

$$
\begin{aligned}
& (g): l t+m u+n v=0, \\
& (p): l t=m u=n v,
\end{aligned}
$$

und welche in der Beziehung zu einander stehen, dafs, wenn man zu den Durchschnittspunkten der Geraden $g$ mit den Coordinatenseiten auf diesen die conjugirten harmonischen Punkte construirt, die Verbindungsgeraden dieser Punkte mit den entsprechenden Gegenecken des Coordinatendreiecks sich im Punkte $p$ durchschneiden: oder man kann auch $g$ als die Axe und $p$ als den Pol zweier homologen Dreiecke definiren, welche als Seiten haben die Coordinatenseiten und die Verbindungsgeraden der Eckpunkte derselben mit den Durchschnittspunkten von $g$ und den correspondirenden Gegenseiten. Der Punkt $p$ soll künftig der Pol der Geraden $g$ in Beziehung auf das Coordinatendreieck*) heifsen.

Ein ähnlicher Zusammenhang findet in der Geometrie des Raumes statt zwischen einer Ebene $\boldsymbol{E}$ und einem Punkte $\boldsymbol{P}$, welche definirt werden durch die Gleichungen:

$$
\begin{aligned}
& (\boldsymbol{E}): l t+m u+n v+p w=0 \\
& (\boldsymbol{P}): l t=m u=n v=p w .
\end{aligned}
$$

*) Der Punkt $p$ ist der Pol der Geraden $g$ in Beziehung auf die durch die Coordinatenseiten gebildete Linie dritten Grades. Vergl. Salmon, Higher plane curves, Art. 155. 
Construirt man zu den Durchschnittsgeraden der Ebene $\boldsymbol{E}$ mit den Coordinatenebenen die Pole in Beziehung auf die entsprechenden Coordinatendreiecke, so ist $\boldsymbol{P}$ der Durchschnittspunkt der Verbindungslinien dieser vier Pole mit den correspondirenden Gegenecken des Coordinatentetraeders; oder auch, wenn man zu den Durchschnittspunkten der Ebene $\boldsymbol{E}$ mit den Seitenkanten des Coordinatentetraeders auf diesen die conjugirten harmonischen Punkte zeichnet, so durchschneiden sich die Verbindungslinien der auf den drei Gegenkantenpaaren liegenden Punkte im Punkte $\boldsymbol{P}$; endlich ist $\boldsymbol{P}$ der Pol und $\boldsymbol{E}$ die Ebene der Homologie zweier homologen Tetraeder (Poncelet, traité des propr. project. Art. 582), nämlich des Coordinatentetraeders und des Tetraeders, dessen Seitenflächen die Verbindungsebenen sind der Eckpunkte des Coordinatentetraeders mit den Durchschnittsgeraden ibrer Gegenflächen und der Ebene $\boldsymbol{E}$. Der Punkt $\boldsymbol{P}$ heifse der $\boldsymbol{P o l}$ der Ebene $\boldsymbol{E}$ in Beziehung auf das Coordinatentetraeder.

Wie bei der geraden Linie einem unendlich entfernten Punkte als conjugirter harmonischer Punkt der Mittelpunkt derselben entspricht, so erhält man in der Ebene als Pol einer unendlich entfernten Geraden den Schwerpunkt des Coordinatendreiecks und im Raume als Pol einer unendlich entfernten Ebene in Beziehung auf das Coordinatentetraeder den Schwerpunkt desselben.

\$. 2.

Dies vorausgesetzt, handle es sich um die Ausdehnung des bekannten Satzes von den Mitten der Diagonalen des vollständigen Vierseits auf den Raum, oder des allgemeineren Satzes: (Man vergleiche Chasles, géom. supérieure, Art. 349.)

Wenn man durch ein vollständiges Vierseit mit seinen drei Diagonalen eine Transversale legt und auf jeder Diagonale zu deren Endpunkten und dem Durchschnittspunkte mit der Transversale die vierten harmonischen Punkte construirt, so liegen diese drei Punkte auf einer geraden Linie.

Man kann die Diagonalen des Vierseits definiren als die Verbindungsgeraden der Eckpunkte eines beliebigen der vier durch die Seiten des Vierseits gebildeten Dreiecke mit den Durchschnittspunkten der Gegenseiten und der jedesmaligen vierten Seite. Dem entsprechend sei das durch drei Seiten 
des Vierseits gebildete Dreieck das Coordinatendreieck, und die Gleichung der vierten Seite:

$$
(x): t+u+v=0,
$$

wo $t, u, v$ beliebige Constanten enthalten, so sind die drei Diagonalen des Vierseits tuvx:

$$
\begin{aligned}
& (u v, t x): \quad u+v=0=t_{1}, \\
& (v t, u x): t \quad+v=0=u_{1}, \\
& (t u, v x): t+u \quad=0=v_{1},
\end{aligned}
$$

woraus sich umgekehrt als Ausdrücke der alten Coordinatenseiten durch die Coordinaten $t_{1}, u_{1}, v_{1}$ ergeben:

$$
2 t=-t_{1}+u_{1}+v_{1}, \quad 2 u=t_{1}-u_{1}+v_{1}, \quad 2 v=t_{1}+u_{1}-v_{1} .
$$

Jetzt sei durch das System die Transversale

$$
(g): \lambda t_{1}+\mu u_{1}+\nu v_{1}=0
$$

gelegt, so ist deren Durchschnittspunkt mit der Diagonale $t_{1}$ :

$$
\left(g, t_{1}\right): t_{1}=0, \quad \mu u_{1}+\nu v_{1}=0,
$$

und als der zugehörige vierte harmonische Punkt in Bezug auf die beiden Endpunkte der Diagonale $t_{1}$ :

und

$$
(t, x): t_{1}=0, \quad u_{1}+v_{1}=0
$$

$$
(u, v): t_{1}=0, \quad u_{1}-v_{1}=0
$$

ergiebt sich der Punkt:

$$
\left(p_{t}\right): t_{1}=0, \quad \frac{u_{1}}{\mu}+\frac{v_{1}}{v}=0
$$

denn setzt man etwa $u_{1}+v_{1}=2 y$ und $u_{1}-v_{1}=2 z$, so werden die Punkte $\left(g, t_{1}\right)$ und $p_{t}$ respective $(\mu+\nu) y+(\mu-\nu) z=0$ und $(\mu+\nu) y-(\mu-v) z=0$, woraus die harmonische Beziehung der vier Punkte zu erkennen ist.

Ebenso ergeben sich als die fraglichen harmonischen Punkte auf den Diagonalen $u_{1}$ und $v_{1}$ respective

$$
\begin{array}{ll}
\left(p_{u}\right): u_{1}=0, & \frac{t_{1}}{\lambda} \quad+\frac{v_{1}}{v}=0 \\
\left(p_{v}\right): v_{1}=0, & \frac{t_{1}}{\lambda}+\frac{u_{1}}{\mu} \quad=0
\end{array}
$$

welche drei Punkte auf der Geraden

$$
\frac{t_{1}}{\lambda}+\frac{u_{1}}{\mu}+\frac{v_{1}}{\nu}=0
$$


liegen: diese Gerade mag die Conjugirte der Geraden $g$ in Beziehung auf das Vierseit tuvx heifsen.

\section{S. 3.}

Ein analoger Satz aus der ebenen Geometrie ist der folgende:

Die Pole einer beliebigen Transversale in Beziehung auf alle Dreiecke, welche eine gemeinsame Spitze haben, und deren Grundlinien in eine und dieselbe gerade Linie fallen, liegen auf einer Geraden.

Der Beweis dieses Satzes, welcher sich leicht aus den harmonischen Beziehungen der Figur ergiebt, läfst sich der späteren Anwendung wegen analytisch, etwa wie folgt, führen. Die Transversale sei:

(1.) $\lambda t+\mu u+v v=0$,

die gemeinsame Grundlinie der Dreiecke:

$$
\boldsymbol{t}+\boldsymbol{u}+\boldsymbol{v}=\mathbf{0}
$$

wo $t, u, v$ beliebige Constanten enthalten mögen, der gemeinsame Eckpunkt der Punkt:

$$
\text { (3.) } t=u=v
$$

und die in demselben zusammentreffenden Seiten von irgend drei Dreiecken:

$$
-2 t+u+v=0, t-2 u+v=0, t+u-2 v=0 .
$$

Um jetzt den Pol zu erhalten der Transversale (1.) in Beziehung auf irgend eines dieser Dreiecke, z. B. welches zu Seiten hat:

$$
t+u+v=0=\dot{t}_{1}, t-2 u+v=0=u_{1}, t+u-2 v=0=v_{1},
$$

nehme man diese Seiten als neue Coordinatenseiten, woraus:

$$
\text { (6.) } 3 t=t_{1}+u_{1}+v_{1}, 3 u=t_{1}-u_{1}, 3 v=t_{1}-v_{1} \text {; }
$$

folglich ergiebt sich als Gleichung der Transversale (1.):

$$
t_{1}(\lambda+\mu+\nu)+u_{1}(\lambda-\mu)+v_{1}(\lambda-\nu)=0
$$

und als Pol dazu:

$$
t_{1}(\lambda+\mu+\nu)=u_{1}(\lambda-\mu)=v_{1}(\lambda-\nu)
$$

oder für die alten Coordinaten :

$$
(t+u+v)(\lambda+\mu+v)=(t-2 u+v)(\lambda-\mu)=(t+u-2 v)(\lambda-v) .
$$

Dieser Pol liegt auf der Geraden:

$$
\text { (8.) } \quad(\mu+\nu) t+(\nu+\lambda) u+(\lambda+\mu) v=0 \text {; }
$$

denn aus den Gleichungen (7.) ergeben sich durch Trennung der Coordinaten 27 * 
die beiden Gleichungen:

und

$$
t(2 \mu+\nu)+u(-\mu+\nu+3 \lambda)+v(2 \mu+\nu)=0
$$

$$
t(2 \nu+\mu)+u(2 \nu+\mu)+v(-\nu+\mu+3 \lambda)=0
$$

und durch deren Addition die Gleichung (8.), d. b. der Pol (7.) liegt auf dieser Geraden. Aber aus der Symmetrie der Gleichung (8.) ergiebt sich, dafs die durch sie dargestellte Gerade zugleich die Pole der Transversale (1.) in Beziehung auf die beiden anderen Dreiecke enthält, was zu beweisen war.

\section{S. 4.}

In der Geometrie des Raumes nunmehr tritt eine erste Analogie des Satzes (\$.2) in gewissen Eigenschaften des allgemeinen Fünfflachs hervor, d. h. des Systems von Figuren, welche sich aus der Durchschneidung von fünf irgend wie im Raume liegenden Ebenen ergeben.

Man kann sich vorstellen, dafs das Fünfflach aus dem Tetraeder hervorgeht durch die Durchschneidung mit einer fünften Ebene; als Diametralebenen ergeben sich dann, wie früher die Diagonalen des Vierseits, diejenigen vier Ebenen, welche die Eckpunkte des Tetraeders mit den Durchschnittsgeraden der fünften Ebene und der jedesmaligen Gegenflächen verbinden. Jede dieser Diametralebenen durchschneidet die Seitenflächen des Tetraeders in vier geraden Linien, von denen immer drei durch einen Punkt, den betreffenden Eckpunkt des Tetraeders, gehen, d. h. welche drei Dreiecke bilden mit gemeinsamer Spitze, und deren Grundlinien auf derselben Geraden liegen. Nennt man diese Dreiecke Diametraldreiecke, so hat man folgenden Satz:

Wenn man durch ein Fünfflach eine Transversalebene legt und in jeder Diametralebene su deren Durchschnitt mit der Transversalebene in Beziehung auf jedes Diametraldreieck den Pol construirt, so liegen die Pole jeder drei Dreiecke derselben Diametralebene auf einer geraden Linie (\$.3). Diese geraden Linien sind zu vier die Generatrices desselben Systems eines Hyperboloids.

Das Fünfflach sei durch die Durchschneidung des Coordinatentetraeders mit der fünften Ebene:

$$
(x): t+u+v+w=0
$$

entstanden, wo wieder die Coordinaten $t, u, v, w$ beliebige Constanten enthalten, so sind die Gleichungen der Diametralebenen des Fünfflachs, d. h. der Ebenen, welche die Eckpunkte des Coordinatentetraeders mit den Durchschnitts- 
geraden der entsprechenden Gegenflächen und der fünften Seitenfläche $x$ verbinden:

$$
\begin{aligned}
& (u v w, t x): \quad u+v+w=0=3 t_{1}, \\
& (v w t, u x): t \quad+v+w=0=3 u_{1}, \\
& (w t u, v x): t+u \quad+w=0=3 v_{1} \\
& (t u v, w x): t+u+v \quad=0=3 w_{1}
\end{aligned}
$$

für diese Ebenen als neue Coordinatenebenen ergeben sich als Ausdrücke der alten Coordinaten:

$$
\begin{array}{rr}
t= & -2 t_{1}+u_{1}+v_{1}+w_{1} \\
u= & t_{1}-2 u_{1}+v_{1}+w_{1} \\
v & =t_{1}+u_{1}-2 v_{1}+w_{1} \\
w & =t_{1}+u_{1}+v_{1}-2 w_{1}
\end{array}
$$

Nunmehr werde als Transversalebene hindurchgelegt die Ebene:

$$
(E): \lambda t_{1}+\mu u_{1}+\nu v_{1}+\widetilde{\omega} w_{1}=0,
$$

so ist deren Durchschnitt mit der Diametralebene $w_{1}$ :

$$
\left(E, w_{1}\right): w_{1}=0, \quad \lambda t_{1}+\mu u_{1}+\nu v_{1}=0 .
$$

Diese Diametralebene aber schneidet aus dem Tetraeder tuvw drei Dreiecke aus, deren Seiten bestimmt sind durch die Durchschneidung mit den Ebenen $u, v, w ; v, t, w ; t, u, w$, d. h. welche für die Coordinaten $t_{1}, u_{1}, v_{1}, w_{1}$ zu Gleichungen haben:

$$
-2 t_{1}+u_{1}+v_{1}=0, \quad t_{1}-2 u_{1}+v_{1}=0, \quad t_{1}+u_{1}-2 v_{1}=0,
$$

und die gemeinsame Grundlinie ist:

$$
t_{1}+u_{1}+v_{1}=0
$$

Nach $\$ .3$ liegen die Pole der Transversale $\left(\boldsymbol{E}, w_{1}\right)$ auf der Geraden

$$
\left(g_{u}\right): w_{1}=0, \quad(\mu+\nu) t_{1}+(\nu+\lambda) u_{1}+(\lambda+\mu) v_{1}=0 .
$$

Nennt man diese Gerade $g_{w}$ die Conjugirte der Transversalebene in Beziehung auf die Diametraldreiecke in $w_{1}$, so erhält man im Ganzen für alle vier Diametralebenen folgendes System von Conjugirten:

$$
\begin{array}{rrrr}
\left(g_{t}\right): t_{1}=0, & (\nu+\widetilde{\omega}) u_{1}+(\widetilde{\omega}+\mu) v_{1}+(\mu+\nu) w_{1}=0 \\
\left(g_{u}\right): u_{1}=0, & (\nu+\widetilde{\omega}) t_{1} & +(\widetilde{\omega}+\lambda) v_{1}+(\lambda+\nu) w_{1}=0 \\
\left(g_{v}\right): v_{1}=0, & (\mu+\widetilde{\omega}) t_{1}+(\widetilde{\omega}+\lambda) u_{1} \\
\left(g_{w}\right): w_{1}=0, & (\mu+\nu) t_{1}+(\nu+\lambda) u_{1}+(\lambda+\mu) v_{1} & =0
\end{array}
$$


Gleichungen, in denen die Coefficienten in Beziehung auf die Diagonale symmetrisch sind, d. h. welche ein System von Generatrices derselben Erzeugungsart eines Hyperboloids darstellen (Siehe später).

Das allgemeine Fünfflach gestattet fünf Gruppirungen seiner Seitenflächen zu vier als Seitenflächen von Tetraedern, je durchschnitten von der fünften Ebene als Transversalebene, und darum ebensoviel Systeme von je vier Diametralebenen, von denen jedoch immer zwei zusammenfallen; der eben bewiesene Satz läfst sich also folgendermafsen verallgemeinern:

In jeder der zehn Diametralebenen des vollstündigen Fünffachs liegen drei Diametraldreiecke und die Pole der Durchschnittsgeraden einer beliebigen Transversalebene mit den einzelnen Diametralebenen in Besiehung auf die zugehörigen Diametraldreiecke je auf einer Geraden, alle dreifsig Pole also unf zehn Geraden. Diese zehn Geraden sind zu vier die Generatrices desselben Systems von fünf verschiedenen Hyperboloiden.

\section{\$. 5.}

Wir gehen jetzt zur Untersuchung eines Sechsflachs über, von welchem drei Paar Ebenen sich in geraden Linien einer und derselben Ebene durchschneiden, und betrachten dasselbe etwa als Hexaeder im engeren Sinne,.. d. h. als Körper mit acht dreikantigen Ecken, jedoch mit erweiterten Seitenflächen, und dessen Gegenflächenpaare in einer (nunmehr aufserhalb des Kernkörpers liegenden) siebenten Ebene das Dreieck $\boldsymbol{A B C}$ ausschneiden. Die Gegenflächenpaare des Kernhexaeders seien:

$$
\begin{aligned}
& A_{1}=A_{1} B_{2} D_{1} C_{2} \text { und } A_{2}=A_{2} B_{1} D_{2} C_{1} \text {, } \\
& \text { - } B_{1}=B_{1} C_{2} D_{1} A_{2} \quad-\quad B_{2}=B_{2} C_{1} D_{2} A_{1} \text {, } \\
& I_{1}^{\prime}=C_{1} A_{2} D_{1} B_{2} \quad-\quad T_{2}=C_{2} A_{1} D_{2} B_{1},
\end{aligned}
$$

von denen sich

$$
A_{1} \text { und } A_{2} \text { in } B C, B_{1} \text { und } B_{2} \text { in } C A, T_{1} \text { und } T_{2} \text { in } A B
$$

durchschneiden mögen. Zieht man jetzt die zwölf Diagonalen in den Seitenflächen des Kernhexaeders, so sind diese die Seitenkanten von zwei Tetraedern $A_{1} B_{1} C_{1} D_{1}$ und $A_{2} B_{2} C_{2} D_{2}$, von denen sich leicht ergiebt, dafs sie homologe Tetraeder sind. Nimmt man nämlich irgend zwei correspondirende Seitendreiecke dieser Tetraeder, z. B. $\boldsymbol{B}_{1} \boldsymbol{C}_{1} \boldsymbol{D}_{1}$ und $\boldsymbol{B}_{2} \boldsymbol{C}_{2} \boldsymbol{D}_{2}$, so durchschneiden sich deren entsprechende Seiten $B_{1} C_{1}$ und $B_{2} C_{2}, C_{1} D_{1}$ und $C_{2} D_{2}, D_{1} B_{1}$ 
$\boldsymbol{D}_{2} \boldsymbol{B}_{2}$ als gerade Linien in den Gegenflächen des Kerntetraeders und, weil sie zu zwei in einer Ebene liegen, resp. in Punkten anf den Seiten $\boldsymbol{C B}$, $A B$ und $C A$ des Dreiecks $A B C$, d. h. die Seitenflächen $B_{1} C_{1} D_{1}$ und $B_{2} C_{2} D_{2}$ selbst durchschneiden sich in einer geraden Linie, welche in der Ebene des Dreiecks $\boldsymbol{A B C}$ liegt. Dasselbe gilt von den drei übrigen Paaren von Seitenflächen der beiden Tetraeder, und diese liégen darum nach der Ponceletschen Erweiterung des Desarguesschen Satzes homolog, d. h. die Verbindungsgeraden der correspondirenden Eckpunkte beider Tetraeder $A_{1} A_{2}, B_{1} B_{2}, C_{1} C_{2}$ und $D_{1} D_{2}$ gehen durch einen und denselben Punkt. Diese Verbindungsgeraden aber sind die Diagonalen des Kerntetraeders: man hat darum folgenden Satz:

Wenn sich die drei Gegenflüchenpaare eines Hexaeders in Geraden einer Ebene durchschneiden, so gehen die vier Diagonalen desselben durch einen und denselben Punkt.

Dieser Durchschnittspunkt der vier Diagonalen sei der Punkt $\boldsymbol{D}$ und werde als vierter Eckpunkt des Tetraeders $\boldsymbol{A B C D}$ genommen, dessen drei Kanten $\boldsymbol{B C}, \boldsymbol{C A}$ und $\boldsymbol{A B}$ die gemeinschaftlichen dritten Diagonalen der Vierseite in den Gegenflächenpaaren $A_{1}$ und $A_{2}, B_{1}$ und $B_{2}, \Gamma_{1}$ und $T_{2}$ sind. Deshalb möge das Tetraeder $A B C D$, sowie die beiden Tetraeder $A_{1} B_{1} C_{1} D_{1}$ und $\boldsymbol{A}_{2} \boldsymbol{B}_{2} \boldsymbol{C}_{2} \boldsymbol{D}_{2}$, deren Kanten die Diagonalen in den Seitenflächen des Kernhexaeders sind, mit dem gemeinsamen Namen Diagonaltetraeder bezeichnet werden.

Diese Benennung rechtfertigt sich auch dadurch, dafs ebenfalls die Kanten $\boldsymbol{A D}, \boldsymbol{B D}, \boldsymbol{C D}$ als Diagonalen gewisser Vierecke auftreten, nämlich zugleich mit zwei Diagonalen der Gegenflächenpaare in den Vierseiten, welche in den Diametralebenen des Hexaeders durch zwei Gegenkanten und zwei Diagonalen des Hexaeders ausgeschnitten werden, z. B. die Kante $\boldsymbol{A D}$ bildet zugleich mit $\boldsymbol{A}_{1} \boldsymbol{D}_{1}$ und $\boldsymbol{A}_{2} \boldsymbol{D}_{2}$ das System der drei Diagonalen des Vierseits, welches in der Diametralebene $A_{1} D_{1} A_{2} D_{2}$ zu aufeinanderfolgenden Seiten die Diagonalen des Hexaeders $A_{1} A_{2}$ und $D_{1} D_{2}$ und die Gegenkanten $A_{1} D_{2}$ und $A_{2} D_{1}$ hat, oder auch $A D, B C$ und $B_{1} C_{1}$ sind die drei Diagonalen des Vierseits, welches in der Diametralebene $B_{1} C_{1} B_{2} C_{2}$ zu aufeinanderfolgenden Seiten die Diagonalen des Hexaeders $\boldsymbol{B}_{1} \boldsymbol{B}_{2}$ und $\boldsymbol{C}_{1} \boldsymbol{C}_{2}$ und die Gegenkanten $\boldsymbol{B}_{1} \boldsymbol{C}_{2}$ und $\boldsymbol{B}_{2} \boldsymbol{C}_{1}$ hat, und ähnlich die beiden andern Kanten $\boldsymbol{B D}$ und $\boldsymbol{C D}$. 
\$. 6.

Nunmehr heifst der Satz vom Hexaeder, dessen vollständige Analogie zu dem von den Diagonalen des Vierseits ( $(\boldsymbol{S} .2)$ nicht zu verkennen ist:

Die Pole einer beliebigen Transversalebene in Beziehung auf die drei Diagonaltetraeder des Hexueders liegen auf einer geraden Linie, oder, wenn die Transversalebene im Unendlichen liegt,

Die Schwerpunkte der drei Diagonaltetraeder des Hexaeders liegen auf einer geraden Linie,

wo also vorausgesetzt ist, dafs sich die Gegenflächenpaare des Hexaeders in Geraden einer Ebene durchschneiden, oder die vier Diagonalen desselben durch einen und denselben Punkt gehen.

Das Sechsflach enthält in seinen sechs Seitenflächen und sechs Diametralebenen zwölf vollständige Vierseite, welche zu zwei eine Diagonale gemeinsam haben: zunächst nämlich die Vierseite der Gegenflächen, deren gemeinschaftliche Diagonalen die Kanten $\boldsymbol{B C}, \boldsymbol{C A}, \boldsymbol{A B}$ sind, dann die Vierseite jeder zwei Diametralebenen, welche in einer der Kanten $\boldsymbol{A D}, \boldsymbol{B D}, \boldsymbol{C D}$ als gemeinsamer Diagonale zusammenstofsen, endlich jede zwei Vierseite einer Seitenfläche und einer Diametralfläche, welche durch Gegenkanten zweier Diagonaltetraeder (z. B. durch die Gegenkanten $B C$ und $A D, B_{1} C_{1}$ und $\left.A_{1} D_{1}\right)$ gehen, also in einer Diagonale einer Seitenfläche des Kernhexaeders $\left(\boldsymbol{A}_{2} \boldsymbol{D}_{2}\right)$ übereinkommen. Legt man jetzt durch das ganze System eine Transversalebene und construirt zu den Durchschnittsgeraden derselben mit den einzelnen Vierseitsebenen die Conjugirten $(\boldsymbol{\$} .2)$, so liegen diese für jede zwei Vierseite, welche eine Diagonale gemeinsam haben, in einer Ebene, weil sie durch denselben Punkt dieser Diagonale gehen müssen, im Ganzen also zu vier in drei Ebenen. Die Conjugirten nämlich jeder vier Vierseite, welche durch Gegenkantenpaare der drei Diagonaltetraeder (z. B. durch $B C$ und $A D, B_{1} C_{1}$ und $A_{1} D_{1}, B_{2} C_{2}$ und $\left.A_{2} D_{2}\right)$ gehen, liegen in einer und derselben Ebene. Jede dieser drei Ebenen mufs alle drei Pole der Transversalebene in Bezug auf die Diagonaltetraeder enthalten, weil jede durch die conjugirten harmonischen Punkte zu den Durchschnittspunkten von Gegenkanten dieser Tetraeder mit der Transversalebene geht (\$. 1.): darum schneiden sich diese Ebenen in einer geraden Linie, auf welcher zugleich die fraglichen Pole selbst liegen müssen. 


\section{\$. 7 .}

Der analytische Beweis des eben entwickelten Satzes giebt zu einer neuen Auffassung der in ihm enthaltenen Resultate und damit zu einer ferneren Analogie unseres Satzes von den Diagonalen des ebenen Vierseits für die Geometrie des Raumes Anlafs.

Die drei Diagonaltetraeder nämlich stehen in der eigenthümlichen Beziehung zu einander, dafs sie zu zwei in vier verschiedenen Anordnungen ihrer Eckpunkte homolog sind, nämlich:

ferner :

$$
\begin{aligned}
& A B C D \text { und } D_{1} C_{1} B_{1} A_{1} \text { mit dem Pol } A_{2}, \\
& A B C D-C_{1} D_{1} A_{1} B_{1}--B_{2} \\
& A B C D-B_{1} A_{1} D_{1} C_{1}--C_{2} \\
& A B C D-A_{1} B_{1} C_{1} D_{1}--D_{2}
\end{aligned}
$$

$A B C D$ und $D_{2} C_{2} B_{2} A_{2}$ mit dem Pol $A_{1}$, $A B C D-C_{2} D_{2} A_{2} B_{2}-\quad-B_{1}$, $A B C D-B_{2} A_{2} D_{2} C_{2}-\quad-C_{1}$ $\boldsymbol{A B C D}-\mathrm{A}_{2} \boldsymbol{B}_{2} \boldsymbol{C}_{2} \boldsymbol{D}_{2}-\quad-\quad-D_{1}$

endlich :

$$
\begin{aligned}
& \boldsymbol{A}_{1} \boldsymbol{B}_{1} \boldsymbol{C}_{1} \boldsymbol{D}_{1} \text { und } \boldsymbol{D}_{2} \boldsymbol{C}_{2} \boldsymbol{B}_{2} \boldsymbol{A}_{2} \text { mit dem Pol } \boldsymbol{A} \text {, } \\
& A_{1} B_{1} C_{1} D_{1}-C_{2} D_{2} A_{2} B_{2}-\quad-B, \\
& A_{1} B_{1} C_{1} D_{1}-B_{2} A_{2} D_{2} C_{2}-\quad-\quad C \text {, } \\
& A_{1} B_{1} C_{1} D_{1}-A_{2} B_{2} C_{2} D_{2}-\quad-\quad-D .
\end{aligned}
$$

Es läfst sich beweisen, dafs die Gegenflächenpaare des Kernhexaeders $A_{1}$ und $A_{2}, B_{1}$ und $B_{2}, \Gamma_{1}$ und $\Gamma_{2}$ respective conjugirte harmonische Ebenen zu den Seitenflächenpaaren des Diagonaltetraeders $\boldsymbol{A B C D}$, welche in den Kanten $B C, C A, A B$ zusammensto/sen, sind. Von diesen Ebenen nämlich enthält jedes Paar des ersten Systems zwei Diagonalen und das entsprechende Paar des zweiten Systems die Endpunkte der dritten Diagonale eines Paars von Diametralvierseiten, z. B. in $A_{1}$ und $A_{2}$ liegen die Diagonalen $A_{1} D_{1}$ und $A_{2} D_{2}$, und $B C D$ und $B C A$ gehen respective durch die Endpunkte $\boldsymbol{D}$ und $\boldsymbol{A}$ der Diagonale $\boldsymbol{D A}$ des Diametralvierecks $\boldsymbol{D A}_{2} \boldsymbol{A D}_{2}$, oder auch in $A_{1}$ und $A_{2}$ liegen respective die Diagonalen $B_{2} C_{2}$ und $B_{1} C_{1}$, und $\boldsymbol{B C D}$ und $\boldsymbol{B C} \boldsymbol{A}$ gehen respective durch die Endpunkte $\boldsymbol{D}$ und $\boldsymbol{A}$ der Diagonale $D A$ des Diametralviereris $D_{B} A C_{1}$, und ähnlich die übrigen. 
Nimmt man darum das Diagonaltetraeder $\boldsymbol{A B C D}$ als Coordinatentetraeder, so lassen sich die Gegenflächenpaare des Hexaeders durch folgende Gleichungen darstellen:

$$
\begin{cases}A_{1}=A_{1} B_{2} D_{1} C_{2}: t+w=0, & \text { und } A_{2}=A_{2} B_{1} D_{2} C_{1}: t-w=0 \\ B_{1}=B_{1} C_{2} D_{1} A_{2}: u+w=0, & \text { und } \quad B_{2}=B_{2} C_{1} D_{2} A_{1}: u-w=0 \\ \Gamma_{1}=C_{1} A_{2} D_{1} B_{2}: v+w=0, & \text { und } \quad \Gamma_{2}=C_{2} A_{1} D_{2} B_{1}: v-w=0\end{cases}
$$

wo $t, u, v, w$ als mit beliebigen Constanten multiplicirt angenommen werden. Es ergeben sich hieraus als Gleichungen der Diametralebenenpaare, welche eine der Kanten $\boldsymbol{A D}, \boldsymbol{B D}, \boldsymbol{C D}$ gemeinschaftlich haben:

$$
\left\{\begin{array}{lll}
B_{1} C_{1} B_{2} C_{2}: u+v=0, & \text { und } & A_{1} D_{1} A_{2} D_{2}: u-v=0 \\
C_{1} A_{1} C_{2} A_{2}: v+t=0, & \text { und } B_{1} D_{1} B_{2} D_{2}: v-t=0 \\
A_{1} B_{1} A_{2} B_{2}: t+u=0, & \text { und } C_{1} D_{1} C_{2} D_{2}: t-u=0
\end{array}\right.
$$

d. h. auch die Diametralebenen sind zu zwei conjugirte harmonische Ebenen in Beziehung auf die in dem Durchschnittspunkt der Diagonalen des Hexaeders zusammensto/senden Seitenflüchen des Diagonaltetraeders $\boldsymbol{A B C D}$.

Die Eckpunkte ferner des Diagonaltetraeders $A_{1} B_{1} C_{1} D_{1}$ erhält man der Reihe nach als Durchschnittspunkte der Ebenensysteme $A_{1}, B_{2}, \Gamma_{2}$; $B_{1}, \Gamma_{2}, A_{2} ; \Gamma_{1}, A_{2}, B_{2} ; A_{1}, B_{1}, \Gamma_{1}$ : ihre Gleichungen sind also:

$$
\text { (3.) }\left\{\begin{array}{rl}
A_{1}:-t=u & v=w \\
B_{1}: t=-u=v \\
C_{1}: t=u \\
D_{1}: t=u
\end{array}\right.
$$

und demnach die Gleichungen ihrer Seitenflächen:

$$
\text { (4.) }\left\{\begin{array}{l}
B_{1} C_{1} D_{1}:-t+u+v+w=0=2 t_{1} \\
C_{1} A_{1} D_{1}: t-u+v+w=0=2 u_{1} \\
A_{1} B_{1} D_{1}: t+u-v+w=0=2 v_{1} \\
A_{1} B_{1} C_{1}: t+u+v-w=0=2 w_{1}
\end{array}\right.
$$

Auf ähnliche Weise erhält man für die Eckpunkte des Diagonaltetraeders $A_{2} B_{2} C_{2} D_{2}$, als Durchschnittspunkte der Ebenensysteme $A_{2}, B_{1}, \Gamma_{1} ; B_{2}, \Gamma_{1}, A_{1}$; $\Gamma_{2}, A_{1}, B_{1} ; A_{2}, B_{2}, \Gamma_{2}$, die Gleichungen: 
Hermes, Satz vom Vierseit auf den Raum ausgedehnt.

$$
\text { (5.) }\left\{\begin{array}{l}
A_{2}: \quad t=-u=-v=w \\
B_{2}:-t=\quad u=-v=w \\
C_{2}:-t=-u=v=w \\
D_{2}: \quad t=\quad u=v
\end{array}\right.
$$

und daraus als Gleichungen ihrer Seitenflächen:

$$
\left\{\begin{array}{l}
B_{2} C_{2} D_{2}: t-u-v+w=0=2 t_{2} \\
C_{2} A_{2} D_{2}:-t+u-v+w=0=2 u_{2} \\
A_{2} B_{2} D_{2}:-t-u+v+w=0=2 v_{2} \\
A_{2} B_{2} C_{2}: t+u+v+w=0=2 w_{2} .
\end{array}\right.
$$

Aus diesen Gleichungen lassen sich leicht die am Anfange dieses Paragraphen gemachten Bemerkungen über die vierfach bomologe Lage der Diagonaltetraeder verificiren, und zugleich ergeben sich die Gleichungen der Ebenen der Homologie selber. Denn zunächst erhält man aus den Gleichungen (4.) als Gleichungen der Ebenen der Homologie für die beiden Tetraeder $\boldsymbol{A B C D}$ und $A_{1} B_{1} C_{1} D_{1}$ oder tuvw und $t_{1} u_{1} v_{1} w_{1}$ in vier verschiedenen Gruppirungen:

$$
\text { (7.) }\left\{\begin{array}{rr}
t-u-v+w= & -t_{1}+u_{1}+v_{1}-w_{1}=0 \\
-t+u-v+w & =t_{1}-u_{1}+v_{1}-w_{1}=0 \\
-t-u+v+w & =t_{1}+u_{1}-v_{1}-w_{1}=0 \\
t+u+v+w & =t_{1}+u_{1}+v_{1}+w_{1}=0
\end{array}\right.
$$

und als die zugehörigen Pole für die Coordinatentetraeder $A B C D$ und $A_{1} B_{1} C_{1} D_{1}$ :

$$
\text { (8.) }\left\{\begin{array}{rlrrr}
t & =-u=-v=w, & \text { oder } & -t_{1}=u_{1}=v_{1}=-w_{1}, \\
-t & =u=-v=w, & \text { oder } & t_{1}=-u_{1}=v_{1}=-w_{1}, \\
-t & =-u=v, & \text { oder } & t_{1}=u_{1}=-v_{1}=-w_{1}, \\
t & =u=v, & \text { oder } & t_{1}=u_{1}=v_{1}=w_{1},
\end{array}\right.
$$

d. h. die einzelnen Seitenflächen und zugehörigen Gegenecken des dritten Diagonaltetraeders $\boldsymbol{A}_{2} \boldsymbol{B}_{2} \boldsymbol{C}_{2} \boldsymbol{D}_{2}$.

Dafs die Ebenen (7.) die Ebenen der Homologie der beiden Tetraeder $A B C D$ und $A_{1} B_{1} C_{1} D_{1}$ sind, folgt aus den Gleichungen (7.) selbst, welche zeigen, dafs jede dieser Ebenen bei dem Uebergange von dem einen Coordinatensystem zum andern sich selbst entspricht. 
Aehnliche Resultate ergeben die Gleichungen (6.), und es läfst sich demnach folgender Satz aussprechen:

Die drei Diagonaltetraeder eines Hexaeders, dessen vier Diagonalen durch einen und denselben Punkt gehen, sind zu zwei in vier verschiedenen Anordnungen ihrer Eckpunkte homolog, und zwar sind für die vier Zusammenstellungen von irgend zwei dieser Tetraeder als homologer Tetraeder die Eckpunkte des dritten Diagonaltetraeders die zugehörigen Pole der Homologie, und die respectiven Gegenflächen desselben die zugehörigen Ebenen der Homologie.

\section{\$.. 8 .}

Betrachten wir jetzt zwei vierfach homologe Tetraeder an und für sich, das eine als Coordinatentetraeder und das zweite durch eins der Systeme von Gleichungen (4.) oder (6.) dargestellt, also etwa die beiden Tetraeder tuvw und $t_{1} u_{1} v_{1} w_{1}$, so ergiebt sich durch die Vergleichung mit den analyti-

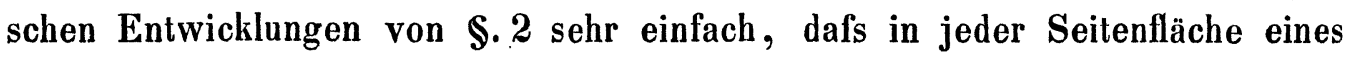
der beiden Tetraeder durch die Seitenflächen des andern ein vollständiges Vierseit ausgeschnitten wird, dessen Diagonalen die Tetraederkanten dieser Seitenfläche selbst sind, z. B. in der Seitenfläche $w$ des Coordinatentetraeders sind die Durchschnittsgeraden mit den Seitenflächen von $t_{1} u_{1} v_{1} w_{1}$ :

$$
\begin{array}{rrr}
w=0, & -t+u+v=0, \\
w=0, & t-u+v=0, \\
w=0, & t+u-v=0, \\
w=0, & t+u+v=0,
\end{array}
$$

d. h. die Seiten eines Vierseits, dessen Diagonalen

$$
w=t=0, \quad w=u=0, \quad w=v=0
$$

sind; legen wir nun durch das System der beiden Tetraeder die Transversalebene

$$
(\boldsymbol{E}): \lambda t+\mu u+\nu v+\widetilde{\omega} w=0,
$$

so ist die zu ihrem Durchschnitt mit der Ebene $w=0$ gehörige Conjugirte (\$.2):

$$
w=0, \quad \frac{t}{\lambda}+\frac{u}{\mu}+\frac{v}{\nu}=0,
$$

und s̊o ergeben sich als die Conjugirten zu den Durchschnitten der Transversalebene $\boldsymbol{E}$ mit allen vier Seitenflächen des Coordinatentetraeders: 
Hermes, Satz vom Vierseit auf den Raum ausgedehnt.

$$
\begin{aligned}
& t=0, \quad \frac{u}{\mu}+\frac{v}{\nu}+\frac{w}{\varpi}=0, \\
& u=0, \frac{t}{\lambda} \quad+\frac{v}{\nu}+\frac{w}{w}=0, \\
& v=0, \frac{t}{\lambda}+\frac{u}{\mu} \quad+\frac{w}{w}=0, \\
& w=0, \frac{t}{\lambda}+\frac{u}{\mu}+\frac{v}{\nu}=0,
\end{aligned}
$$

Linien, welche in der Ebene

$$
\frac{t}{\lambda}+\frac{u}{\mu}+\frac{v}{\nu}+\frac{w}{w}=0
$$

liegen, d. h.:

Die Seitenflächen eines Tetraeders werden durch die eines ihm vierfach homologen Tetraeders in vier Vierseiten durchschnitten, welche in der Beziehung zu einander stehen, da/s die Conjugirten der Durchschnittsgeraden einer beliebigen Transversalebene mit den Seitenflächen des ersten Tetraeders in Bezug auf diese Vierseite in derselben Ebene liegen.

Berlin, im October 1858. 